ARTICLE

\title{
Constructing chiral bicyclo[3.2.1]octanes via palladium-catalyzed asymmetric tandem Heck/ carbonylation desymmetrization of cyclopentenes
}

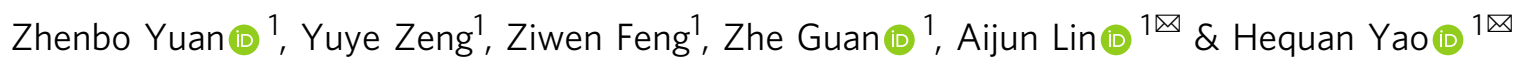

Transition-metal-catalyzed tandem Heck/carbonylation reaction has emerged as a powerful tool for the synthesis of structurally diverse carbonyl molecules, as well as natural products and pharmaceuticals. However, the asymmetric version was rarely reported, and remains a challenging topic. Herein, we describe a palladium-catalyzed asymmetric tandem Heck/ carbonylation desymmetrization of cyclopentenes. Alcohols, phenols and amines are employed as versatile coupling reagents for the construction of multifunctional chiral bicyclo [3.2.1] octanes with one all-carbon quaternary and two tertiary carbon stereogenic centers in high diastereo- and enantioselectivities. This study represents an important progress in both the asymmetric tandem Heck/carbonylation reactions and enantioselective difunctionalization of internal alkenes.

\footnotetext{
${ }^{1}$ State Key Laboratory of Natural Medicines (SKLNM) and Department of Medicinal Chemistry, School of Pharmacy, China Pharmaceutical University,

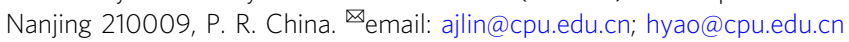


T ransition-metal (TM)-catalyzed carbonylation reaction ${ }^{1-16}$, especially palladium-catalyzed tandem Heck/carbonylation reaction, presents an efficient method to construct a variety of synthetically versatile carbonyl compounds from readily accessible organic halides and alkenes ${ }^{17-21}$. Moreover, these methods have been applied as key steps in the total synthesis of natural products and bioactive molecules ${ }^{22-28}$. Very recently, Reisman and co-workers realized the total synthesis of $(+)$-Perseanol employing palladium-catalyzed tandem Heck/carbonylation to assemble the vital tetracyclic core (Fig. 1a) ${ }^{29}$. However, the asymmetric version of tandem Heck/carbonylation reactions was rare, and remains a challenging topic. Some inherent issues, such as the strong $\pi$-acidity and coordination ability of $\mathrm{CO}$, would hamper the oxidative addition of organohalides towards low-valent metal species ${ }^{30}$. In addition, the harsh reaction conditions (high temperature and high CO pressure), the incidental racemization $^{31}$, the $\beta$-hydrogen elimination of alkylpalladium intermediates, the direct carbonylation of organohalides, and other competitive side-reactions make the asymmetric progress more difficult and complicated. Recently, three representative works on palladium-catalyzed asymmetric tandem Heck/carbonylation reaction of 1,1-disubstitueted alkenes to synthesize dihydrobenzofurans, oxindole derivatives, and 3,4-dihydroisoquinolines have been realized by Correia's group ${ }^{32}$, Zhu and Luo's group ${ }^{33}$, and Zhang's group ${ }^{34}$, respectively. In contrast to the success of 1,1-disubstituted alkenes (the alkylpalladium intermediates lack eliminable $\beta$-hydrogen), the TM-catalyzed asymmetric tandem Heck/carbonylation reaction of unactivated internal alkenes has not been developed until now.

On the other hand, bicyclo[3.2.1] octanes are found in several natural products with antibacterial, antioxidant, antithrombosis, and antitumor activities ${ }^{35,36}$. However, constructing such intricate polycyclic bridge ring compounds with multiple chiral centers simultaneous implementation remains a challenging project $^{37-46}$. Based on our research interest in Heck reactions ${ }^{47-50}$, herein, we describe a palladium-catalyzed asymmetric tandem Heck/carbonylation desymmetrization of cyclopentenes to construct multifunctional chiral bicyclo[3.2.1] octanes bearing one allcarbon quaternary and two tertiary carbon stereogenic centers in excellent diastereoselectivities and enantioselectivities (Fig. 1b). Suppressing the foreseeable side reactions, such as $\beta$-hydrogen elimination of alkylpalladium intermediates, and the direct $\mathrm{CO}$ insertion or nucleophiles insertion reaction (Fig. 1c), is the key to the success of this reaction.

\section{Results}

Reaction optimization. After systematic evaluation of the reaction conditions, the desired chiral bicyclo[3.2.1]octane product 3aa was achieved in $81 \%$ yield and $96 \%$ ee employing $\mathrm{Pd}_{2} \mathrm{dba}_{3} \cdot \mathrm{CHCl}_{3}(5 \mathrm{~mol} \%)$ as the catalyst, (S)-Difluorphos L1 $(20$ $\mathrm{mol} \%$ ) as the ligand, $\mathrm{K}_{2} \mathrm{CO}_{3}$ (2 equiv) as the base, and mixed 1,2-

A Palladium-catalyzed tandem Heck/carbonylation in total synthesis of Perseanol (Reisman et al., Nature, 2019)

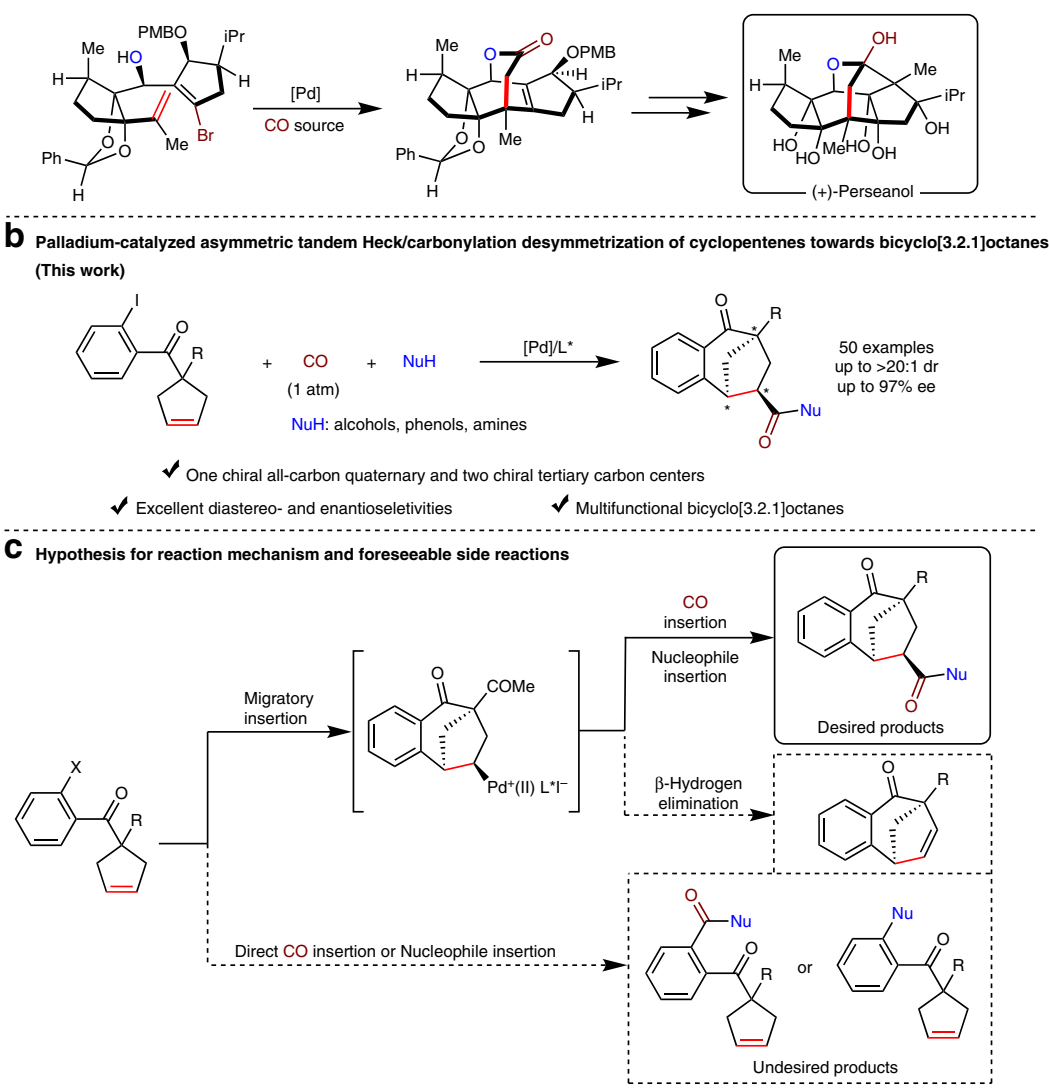

Fig. 1 Palladium-catalyzed tandem Heck/carbonylation reactions of alkenes. a Pd-catalyzed tandem Heck/carbonylation as key step to the total synthesis of (+)-Perseanol. b Palladium-catalyzed asymmetric tandem Heck/carbonylation of internal alkenes towards bicyclo[3.2.1]octanes (this work). c Hypothesis of mechanism for the tandem Heck/carbonylation reaction and foreseeable side reactions. 


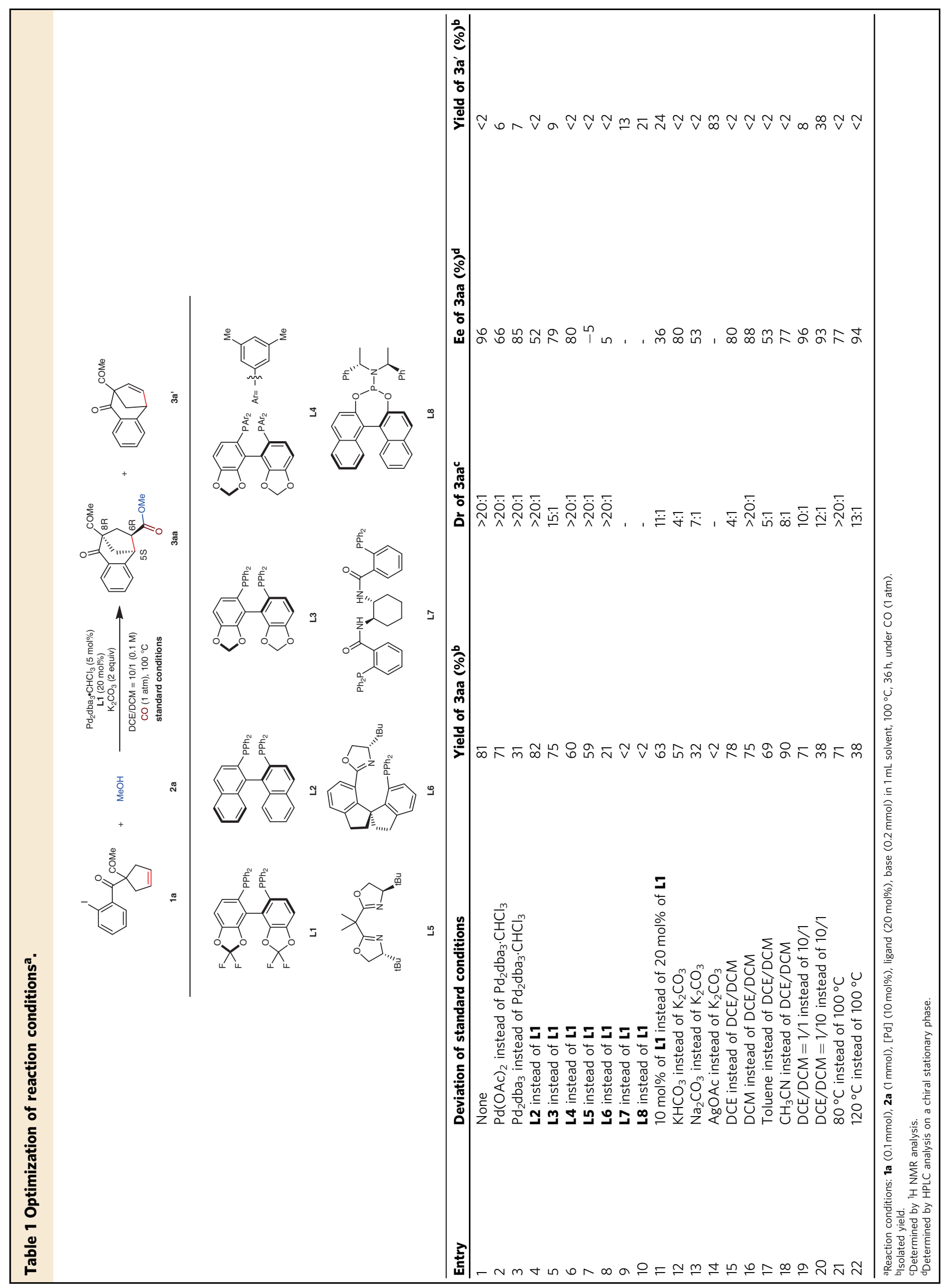


dichloroethane (DCE)/dichloromethane (DCM) (10/1) as the solvent at $100{ }^{\circ} \mathrm{C}$ (Table 1 , entry 1$)$. Other catalysts, such as $\mathrm{Pd}$ $(\mathrm{OAc})_{2}$ and $\mathrm{Pd}_{2}(\mathrm{dba})_{3}$ were less effective (entries 2 and 3). BINAP L2, SEGPHOS L3, DM-SEGPHOS L4 offered 3aa in $52-80 \%$ ee (entries 4-6), while BOX-type ligand L5, PHOX-type ligand L6, bis(phosphine-amide) ligand L7 and phosphoramidite ligand L8 caused the reaction inactivation (entries 7-10). Decreasing the amount of ligand resulted 3aa in diminished yield, diastereo- and enantioselectivity (entry 11). Screening the additives revealed that $\mathrm{K}_{2} \mathrm{CO}_{3}$ was optimal, and AgOAc delivered racemic $\mathbf{3} \mathbf{a}^{\prime}$ in $83 \%$ yield, which was formed via $\beta$-hydrogen elimination (entries 1 and 12-14). The choice of solvent, also the ratio of the mixed solvent, was crucial to the reaction efficiency (entries 15-20). Adjusting the reaction temperature was inconducive to improve the outcome of the reaction (entries 21-22). The structure and absolute configuration of 3aa were confirmed by single-crystal Xray diffraction analysis (see the Supplementary Note 3 for details).

Substrate scope. With the optimized reaction conditions in hand, we then tested the substrate scope of alcohols in this asymmetric Heck/carbonylation reaction, and the results were summarized in Fig. 2. Simple primary alcohols, such as ethanol, $n$-propanol and benzyl alcohol afforded the products 3aa-ad in moderate to good yields with high enantioselectivities. It is noted that aryl bromine derivative was also a good candidate, delivering $\mathbf{3 a b}$ in $50 \%$ yield with $94 \%$ ee after prolonging the time to $48 \mathrm{~h}$. Besides, other primary alcohols with various functional groups, such as alkenyl, trifluoromethyl, halogen, trimethylsilyl, even highly sterically hindered adamantly group, all performed well, offering $\mathbf{3 a e - a i}$ in 88-96\% ee. Cyclic and acyclic secondary alcohols delivered the corresponding products $\mathbf{3 a j - a o}$ in good efficiency. Products $\mathbf{3 b a - d a}$ with different substituents on the benzene ring were produced in good yields with high enantioselectivities. Product 3eb with two ester groups was achieved in $68 \%$ yield with $95 \%$ ee.

Phenol esters are important skeletons in pharmaceuticals and bioactive compounds. Although phenols as nucleophilic reagents have been employed in some carbonylation reactions ${ }^{51,52}$, they have not met with the success in asymmetric tandem Heck/ carbonylation reactions, because the two potential nucleophilic sites at $\mathrm{O}$ and $\mathrm{C}$ of phenols would increase the complexity of the reaction. Herein, phenols as versatile components were performed in our asymmetric Heck/carbonylation reactions with $\mathrm{KHCO}_{3}$ as the base and toluene as the solvent (Fig. 3). Electron donating groups $(-\mathrm{Me},-t \mathrm{Bu}$, and $-\mathrm{OMe})$, a halogen group $(-\mathrm{Cl})$, an electron withdrawing group $(-\mathrm{COMe})$, as well as a phenyl group at the para-position of phenols offered the corresponding products $5 \mathbf{a a}-\mathbf{a g}$ in $91-95 \%$ ee. meta-Chlorine
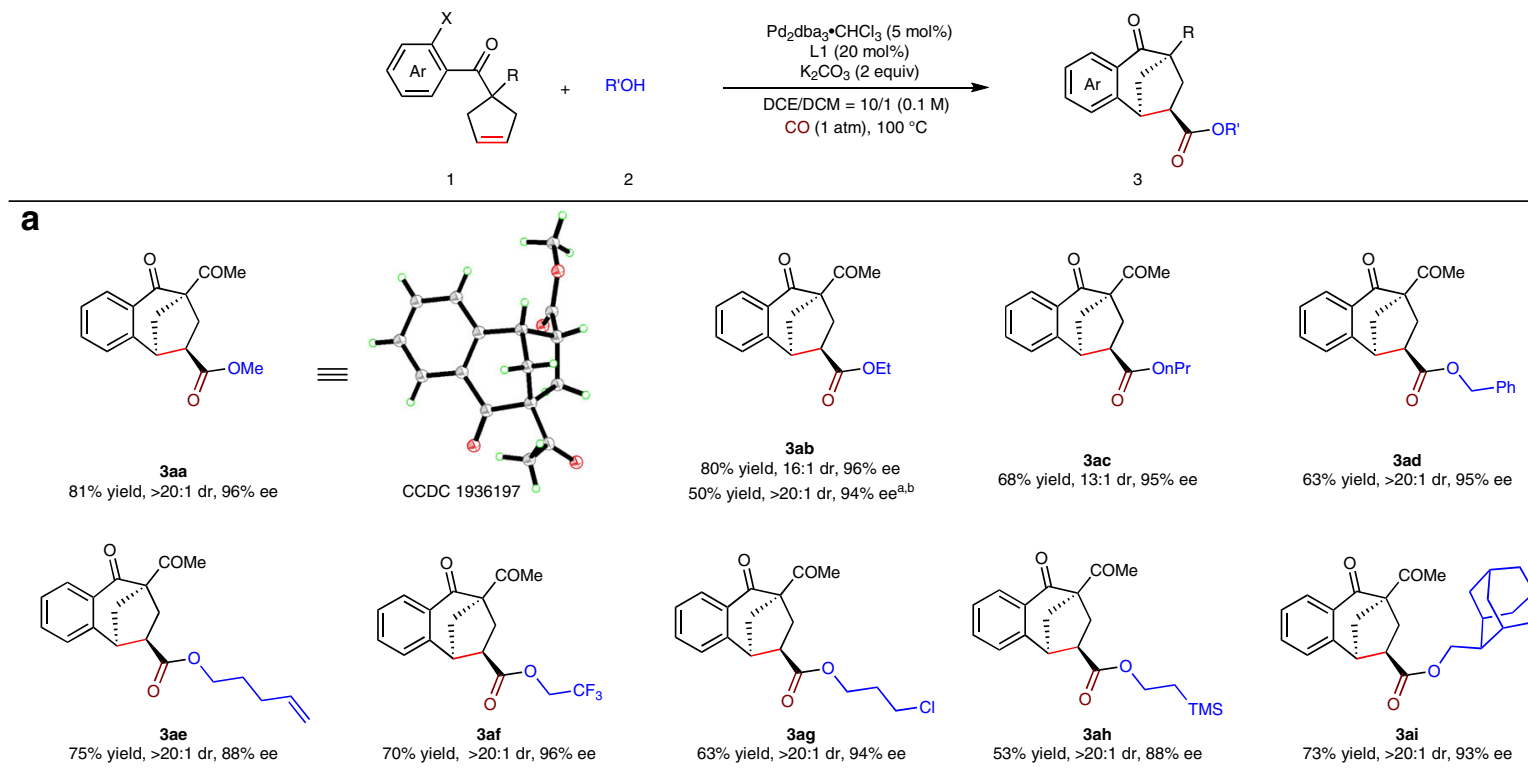

3ac
$68 \%$ yield, $13: 1 \mathrm{dr}, 95 \%$ ee

3ad
$63 \%$ yield, $>20: 1 \mathrm{dr}, 95 \%$ ee

b
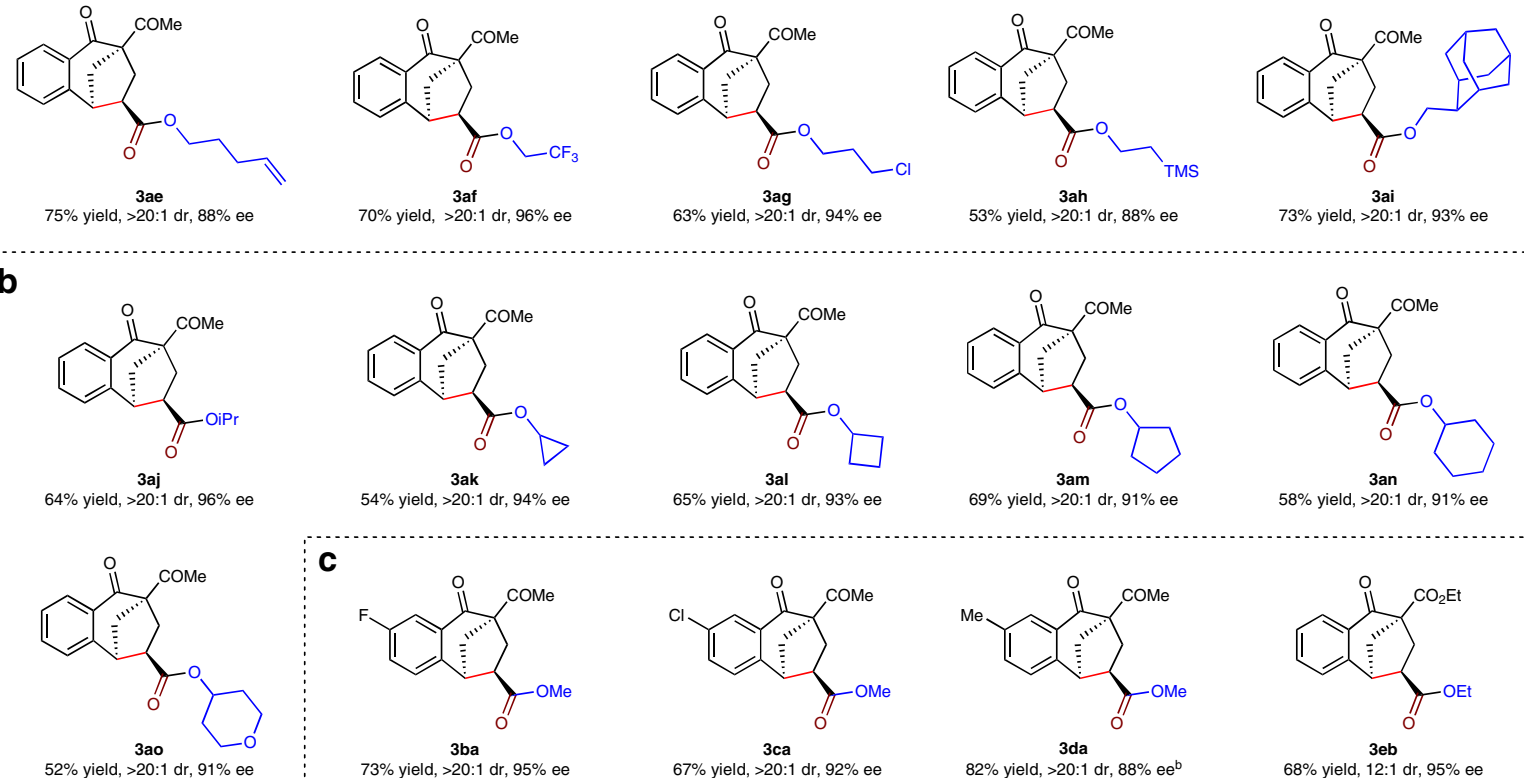

3ah
$53 \%$ yield, $>20: 1 \mathrm{dr}, 88 \%$ ee

3ai 73 yield, $>20: 1 \mathrm{dr}, 93 \%$ ee
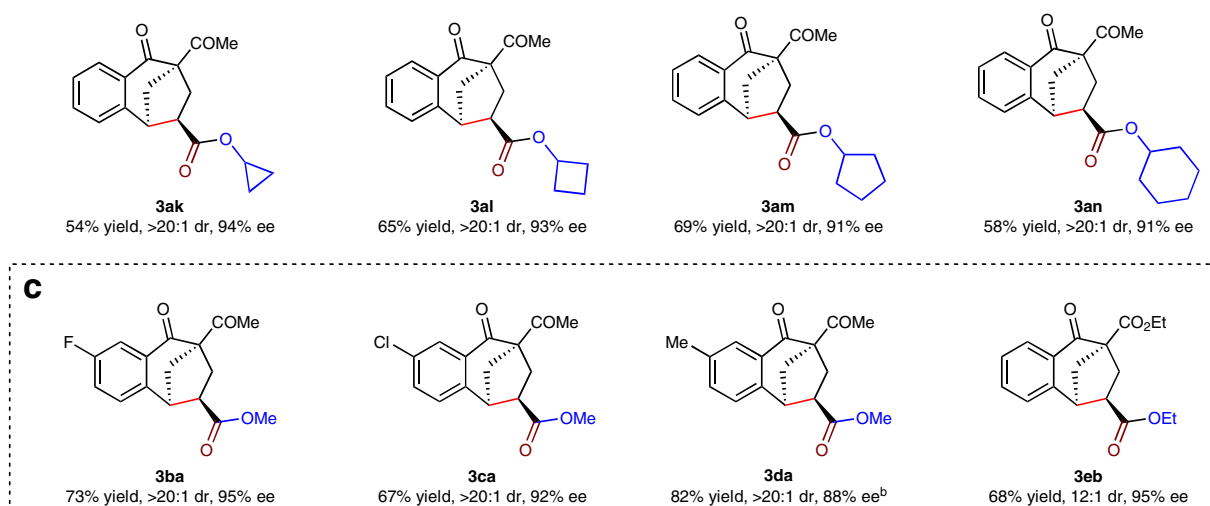

Fig. 2 Substrate scope. a Substrate scope of primary alcohols. b Substrate scope of secondary alcohols. c Substrate scope of the benzoylcyclopentenes. Reaction conditions: $X=\mathrm{I}, \mathbf{1}(0.1 \mathrm{mmol}), \mathbf{2}(1 \mathrm{mmol}), \mathrm{Pd}_{2} \mathrm{dba}_{3} \bullet \mathrm{CHCl}_{3}(5 \mathrm{~mol} \%), \mathbf{L 1}(20 \mathrm{~mol} \%), \mathrm{K}_{2} \mathrm{CO}_{3}(0.2 \mathrm{mmol})$ in $1 \mathrm{~mL}$ solvent, $100^{\circ} \mathrm{C}, 36 \mathrm{~h}$, under $\mathrm{CO}$ (1 atm). Yields of isolated products are given. The $\mathrm{dr}$ values were determined by ${ }^{1} \mathrm{H}$ NMR analysis. The ee values were determined by $\mathrm{HPLC}$ analysis on a chiral stationary phase. ${ }^{a} \mathrm{X}=\mathrm{Br}$. ${ }^{\mathrm{b}} 48 \mathrm{~h}$. 


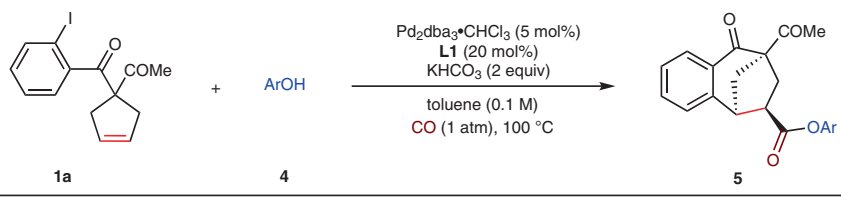

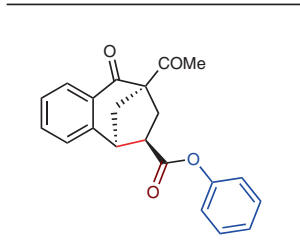

$\stackrel{5 a a}{71 \% \text { yield, }>20: 1 \mathrm{dr}, 94 \% \text { ee }}$
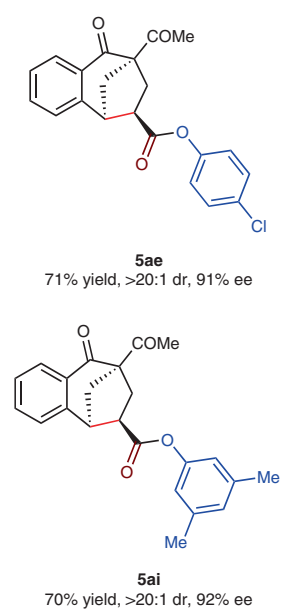

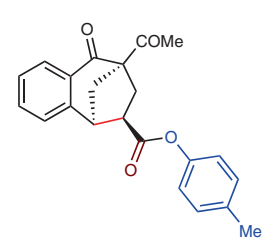

$\stackrel{5 a b}{77 \% \text { yield, }>20: 1 \mathrm{dr}, 95 \% \text { ee }}$

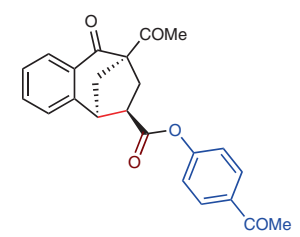

$\stackrel{5 \text { af }}{50 \% \text { yield, }>20: 1} \mathrm{dr}, 93 \%$ ee

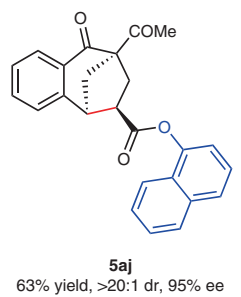

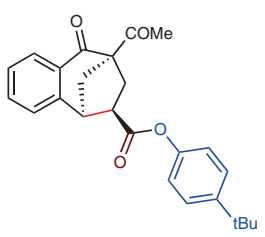

$\underset{76 \% \text { yield, }>20: 1 \mathrm{dr}, 92 \% \text { ee }}{5 \mathrm{ac}}$

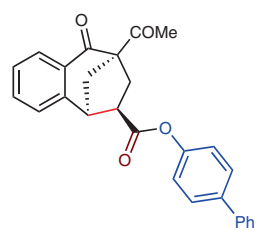

5ag
$69 \%$ yield, $>20: 1 \mathrm{dr}, 94 \%$ ee

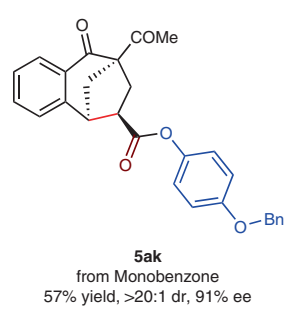

$57 \%$ yield, $>20: 1 \mathrm{dr}, 91 \%$ ee

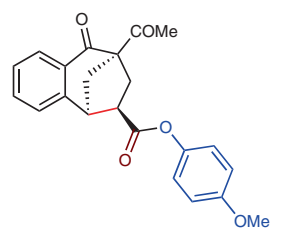

$\stackrel{5 a d}{55 \% \text { yield, }>20: 1 \mathrm{dr}, 93 \% \text { ee }}$

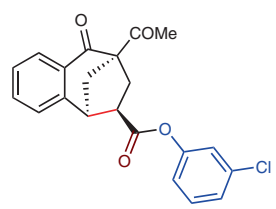

$\underset{54 \% \text { yield, }>20: 1 \mathrm{dr}, 95 \% \text { ee }}{5 \text { ah }}$

Fig. 3 Substrate scope of phenols. Reaction conditions: 1a $(0.1 \mathrm{mmol}), \mathbf{4}(0.25 \mathrm{mmol}), \mathrm{Pd}_{2} \mathrm{dba}_{3} \bullet \mathrm{CHCl}_{3}(5 \mathrm{~mol} \%), \mathbf{L 1}(20 \mathrm{~mol} \%), \mathrm{KHCO} 3(0.2 \mathrm{mmol})$ in toluene $(1 \mathrm{~mL}), 100{ }^{\circ} \mathrm{C}, 36 \mathrm{~h}$, under $\mathrm{CO}(1 \mathrm{~atm})$. Yields of isolated products are given. The $\mathrm{dr}$ values were determined by ${ }^{1} \mathrm{H}$ NMR analysis. The ee values were determined by HPLC analysis on a chiral stationary phase.

substituted phenol $\mathbf{4 h}$ and 3,5-dimethylphenol $\mathbf{4 i}$ could also fulfill the reaction well, and no significant steric hindrance effect was observed. 1-Naphthol delivered 5aj in 95\% ee. Moreover, monobenzone, as a potent skin lightener drug, could give the adduct 5 ak in $91 \%$ ee.

To further exhibit the robustness and generality of this reaction, scope of nitrogen nucleophiles was investigated with $\mathrm{Pddba}_{2}(10 \mathrm{~mol} \%)$ as the catalyst, $\mathbf{L 1}(20 \mathrm{~mol} \%)$ as the ligand, $\mathrm{K}_{2} \mathrm{HPO}_{4}(0.2 \mathrm{mmol})$ as the base in $\mathrm{CH}_{3} \mathrm{CN}(1 \mathrm{~mL})$ at $100{ }^{\circ} \mathrm{C}$ (Fig. 4). Acyclic secondary alkylamines, such as diethylamine and dibenzylamine delivered products 7 aa (see the Supplementary Note 4 for details on the X-ray crystal structure) and 7ab in 93 and $94 \%$ ee. Cyclic secondary alkylamines furnished products 7ac-ag in $92-94 \%$ ee. Alkylarylamines, such as $N$-methylaniline and indoline, provided 7ah and 7ai in 92 and $91 \%$ ee. Primary alkylamines, such as $n$-propylamine, benzylamines, and thiophenylmethanamine offered products 7aj-am in $92-94 \%$ ee. Primary arylamines were also qualified to work in this reaction, delivering products 7an-ap in good enantioselectivities with $\mathrm{KHCO}_{3}$ as the base after prolonging the reaction time to $48 \mathrm{~h}$. 5-OMesubstituted cyclopentene 1f performed smoothly to give $\mathbf{7 f b}$ in 97\% ee. Finally, pharmaceuticals including Vortioxetine, Trimetazidine and Riluzole were all well late-stage functionalized with bicyclo[3.2.1] octanes to offer $7 \mathbf{a q}-$ as in $88-95 \%$ ee.

Further study. The enantiodivergent synthesis of $(5 R, 6 S, 8 S)-5 \mathbf{a c}^{\prime}$ was also realized in $70 \%$ yield and $97 \%$ ee employing $(R)$ Difluorphos as the ligand (Fig. 5a). To demonstrate the mechanism of this reaction, study on nonlinear effect of the enantioselectivity of 5ac was carried out (Fig. 5b). The linear correlation $\left(R^{2}=0.99\right)$ between the enantioselectivities of the product $5 \mathbf{a c}$ and the enantiopurities of the ligand $\mathbf{L} \mathbf{1}$ revealed the involvement of one active catalyst species in the stereodetermining transition states of the migratory insertion process. On the basis of the above-mentioned results and previous literatures $31,32,47$, a proposed mechanism of this reaction is figured in Fig. 5c. Firstly, oxidative addition of the active palladium catalyst with $\mathbf{1 a}$ delivers the cationic $\operatorname{Pd}(\mathrm{II})$ intermediate $\mathbf{I}$. Intramolecular syn-migratory insertion of I results in the alkylpalladium intermediate II, which followed by the insertion of $\mathrm{CO}$ delivers the intermediate III. Finally, the nucleophile insertion of the phenol $\mathbf{4 c}$ to the intermediate III produces the product 5ac. It is noted that the high diastereoselectivity was arisen from the stereospecific syn-migratory insertion step, which has been confirmed in our previous work by the deuterium-labeling experiments ${ }^{47}$. The observed stereochemical outcome of the reaction with the C2-symmetric, $(S)$-configured ligand L1 can be rationalized based on the two diastereomeric intermediates A1 and B1 (Fig. 5d). The transition state $\mathbf{B} \mathbf{1}$ is notable for the severe steric repulsion between the benzoyl moiety of the cyclopentene $1 \mathbf{a}$ and the benzene ring of the ligand $\mathbf{L 1}$, a factor which is not present in the transition state A1; this may account for the predominance of the $(5 S, 6 R, 8 R)$ enantiomer of $5 \mathbf{a c}$ in the product.

\section{Discussion}

In summary, we have developed a Pd-catalyzed asymmetric tandem Heck/carbonylation desymmetrization of cyclopentenes. Various bicyclo[3.2.1] octanes bearing one chiral all-carbon 

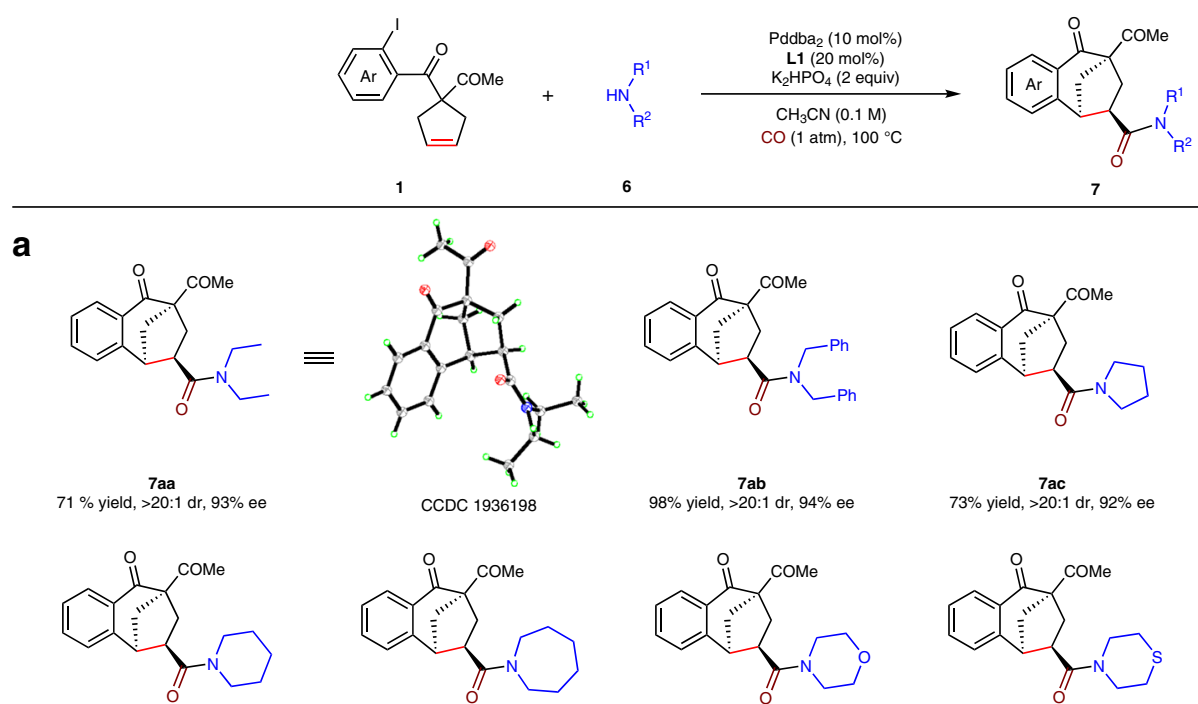

$7 \mathrm{ab}$
$98 \%$ yield, $>20: 1 \mathrm{dr}, 94 \%$ ee

7ac
$73 \%$ yield, $>20: 1 \mathrm{dr}, 92 \%$ ee
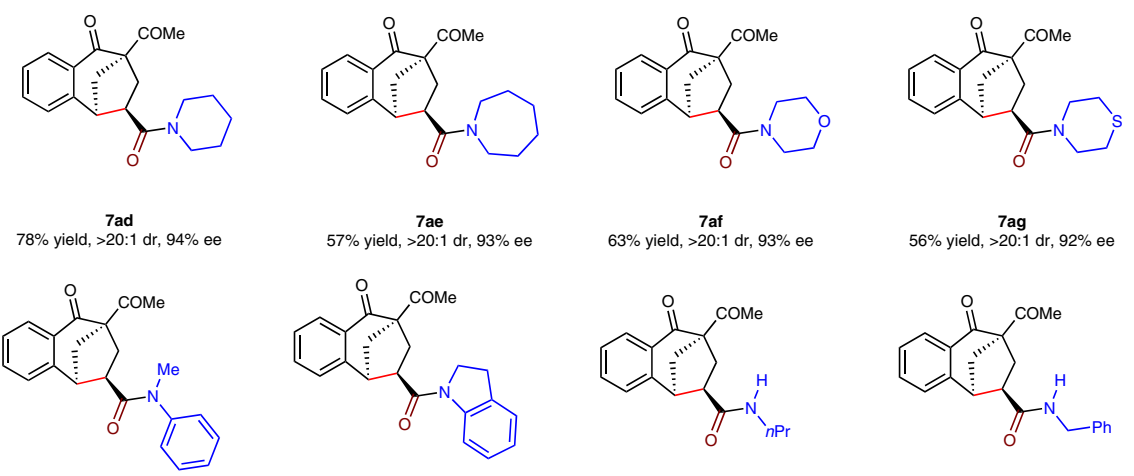

$\underset{57 \% \text { yield, }>20: 1 \mathrm{dr}, 93 \% \text { ee }}{ }$

7af
$63 \%$ yield, $>20: 1 \mathrm{dr}, 93 \%$ ee

$\underset{56 \% \text { yield, }>20: 1 \mathrm{dr}, 92 \% \text { ee }}{\stackrel{7 a g}{2}}$
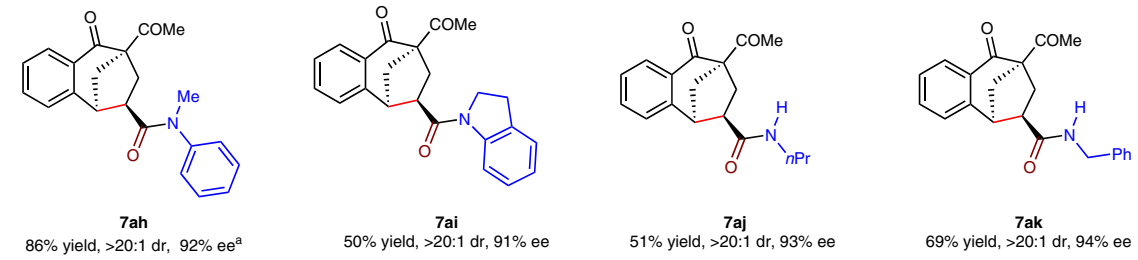

$50 \%$ yield, $>20: 1 \mathrm{dr}, 91 \%$ ee

$\stackrel{7 \text { aj }}{51 \% \text { yield, }>20: 1 \mathrm{dr}, 93 \% \text { ee }}$

7ak
$69 \%$ yield, $>20: 1 \mathrm{dr}, 94 \%$ ee<smiles></smiles>
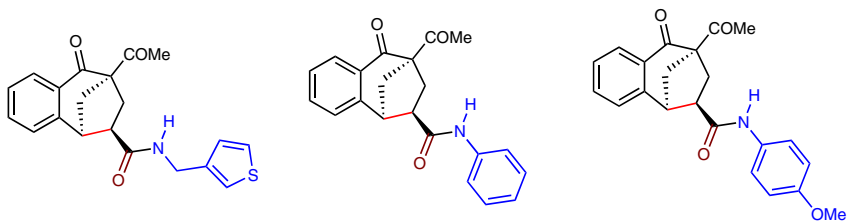

7al
$58 \%$ yield, $>20: 1 \mathrm{dr}, 92 \%$ ee

$7 \mathrm{am}$
$61 \%$ yield, $>20: 1 \mathrm{dr}, 93 \%$ ee

7 an

77\% yield, $>20: 1 \mathrm{dr}, 93 \% \mathrm{ee}^{\mathrm{a}}$ $7 \mathrm{ao}$
$86 \%$ yield, $>20: 1 \mathrm{dr}, 93 \% \mathrm{ee}^{\mathrm{a}}$<smiles>CCOc1ccc(NC(=O)C2C[C@@]3(C(C)=O)C[C@H]2c2ccccc2C3=O)cc1</smiles><smiles>COc1ccc2c(c1)C(=O)C(C)(C(C)=O)CC2C(=O)N(Cc1ccccc1)Cc1ccccc1</smiles>

b<smiles>Cc1ccc(Sc2ccccc2N2CCN(C(=O)C3CC(C)C4CC3c3ccccc3C4=O)CC2)c(C)c1</smiles>

7 aq from Vortioxetine
$50 \%$ yield, $>20: 1 \mathrm{dr}, 94 \%$ ee<smiles>COc1ccc(CN2CCN(C(=O)[C@@H]3C[C@@H]4C[C@H]3c3ccccc3C4=O)CC2)c(OC)c1OC</smiles>
$55 \%$ yield, $>20: 1 \mathrm{dr}, 88 \%$<smiles>CC(=O)[C@]12C[C@H](C(=O)Nc3nc4ccc(OC(F)(F)F)cc4s3)[C@@H]3C[C@H](c4ccccc4C3=O)[C@H]1C2</smiles>

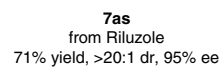

Fig. 4 Substrate scope. a Substrate scope of amines. $\mathbf{b}$ Application to asymmetric late-stage functionalization of pharmaceuticals. Reaction conditions: 1a $(0.1 \mathrm{mmol}), \mathbf{6}(0.2 \mathrm{mmol}), \mathrm{Pddba}_{2}(10 \mathrm{~mol} \%), \mathbf{L 1}(20 \mathrm{~mol} \%), \mathrm{K}_{2} \mathrm{HPO}_{4}(0.2 \mathrm{mmol})$ in $\mathrm{CH}_{3} \mathrm{CN}(1 \mathrm{~mL}), 100{ }^{\circ} \mathrm{C}, 36 \mathrm{~h}$, under $\mathrm{CO}(1 \mathrm{~atm})$. Yields of isolated products are given. The dr values were determined by ${ }^{1} \mathrm{H}$ NMR analysis. The ee values were determined by HPLC analysis on a chiral stationary phase. ${ }^{a} \mathrm{~K}_{2} \mathrm{HPO}_{4}$ was replaced by $\mathrm{KHCO}_{3}, 48 \mathrm{~h}$. b48 h.

quaternary and two tertiary carbon stereogenic centers were obtained in moderate to good yields with excellent diastereoselectivities and enantiomeric excess. This method provided a general and practical route for the enantioselective difunctionalization of unactivated internal alkenes and chiral bicyclo[3.2.1] octanes.

\section{Methods}

General procedure for the catalytic reactions with alchohols. A sealed tube was charged with the cyclopentenes $\mathbf{1}\left(0.1 \mathrm{mmol}, 1\right.$ equiv), $\mathrm{Pd}_{2} \mathrm{dba}_{3} \cdot \mathrm{CHCl}_{3}(5 \mathrm{~mol} \%)$, L1 $(20 \mathrm{~mol} \%)$, and $\mathrm{K}_{2} \mathrm{CO}_{3}(0.2 \mathrm{mmol}, 2$ equiv). The vial was thoroughly flushed with CO, and alchohols 2 ( $1 \mathrm{mmol}, 10$ equiv), as well as DCE/DCM $(10 / 1,1 \mathrm{~mL})$ were added under $\mathrm{CO}$ atmosphere. The reaction mixture was stirred at $100^{\circ} \mathrm{C}$ for $36 \mathrm{~h}$. After the reaction vessel was cooled to room temperature, the solution was concentrated in vacuo and purified by careful chromatography on silica gel $(200-300 \mathrm{mesh})(\mathrm{PE} / \mathrm{EA}=4 / 1)$ to afford the desired products 3 .
General procedure for the catalytic reactions with phenols. A sealed tube was charged with the cyclopentenes $1(0.1 \mathrm{mmol}, 1$ equiv), phenols $4(0.25 \mathrm{mmol}, 2.5$ equiv), $\mathrm{Pd}_{2} \mathrm{dba}_{3} \cdot \mathrm{CHCl}_{3}$ (5 mol\%), $\mathbf{L l}(20 \mathrm{~mol} \%)$, and $\mathrm{KHCO}_{3}(0.2 \mathrm{mmol}, 2$ equiv). The vial was thoroughly flushed with $\mathrm{CO}$, and toluene $(1 \mathrm{~mL})$ was added under $\mathrm{CO}$ atmosphere. The reaction mixture was stirred at $100{ }^{\circ} \mathrm{C}$ for $36 \mathrm{~h}$. After the reaction vessel was cooled to room temperature, the solution was concentrated in vacuo and purified by careful chromatography on silica gel $(200-300$ mesh) $(\mathrm{PE} / \mathrm{EA}=4 / 1)$ to afford the desired products 5 .

General procedure for the catalytic reactions with amines. A sealed tube was charged with the cyclopentenes 1 ( $0.1 \mathrm{mmol}, 1$ equiv), $\mathrm{Pddba}_{2}$ ( $\left.10 \mathrm{~mol} \%\right)$, L1 (20 mol\%), and $\mathrm{K}_{2} \mathrm{HPO}_{4}(0.2 \mathrm{mmol}, 2$ equiv). The vial was thoroughly flushed with $\mathrm{CO}$, and amines $6\left(0.2 \mathrm{mmol}, 2\right.$ equiv), as well as $\mathrm{CH}_{3} \mathrm{CN}(1 \mathrm{~mL})$ were added under $\mathrm{CO}$ atmosphere. The reaction mixture was stirred at $100^{\circ} \mathrm{C}$ for $36 \mathrm{~h}$. After the reaction vessel was cooled to room temperature, the solution was concentrated in vacuo and purified by careful chromatography on silica gel (200-300 mesh) (PE/ $\mathrm{EA}=2 / 1)$ to afford the desired products 7 . 
a

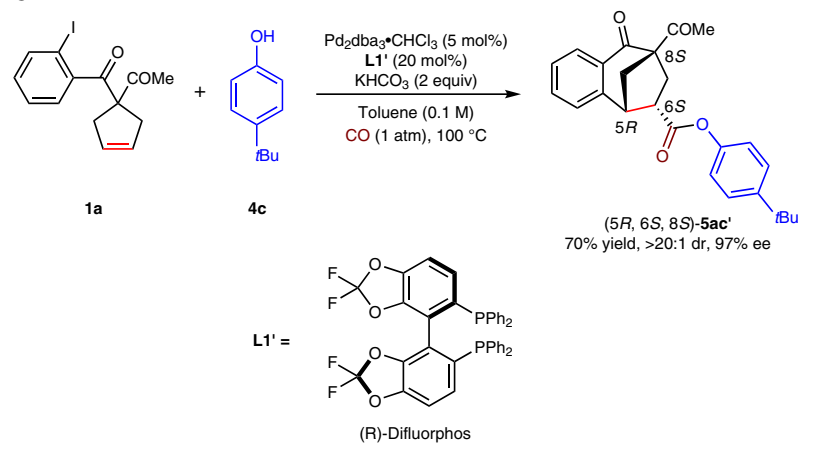

C

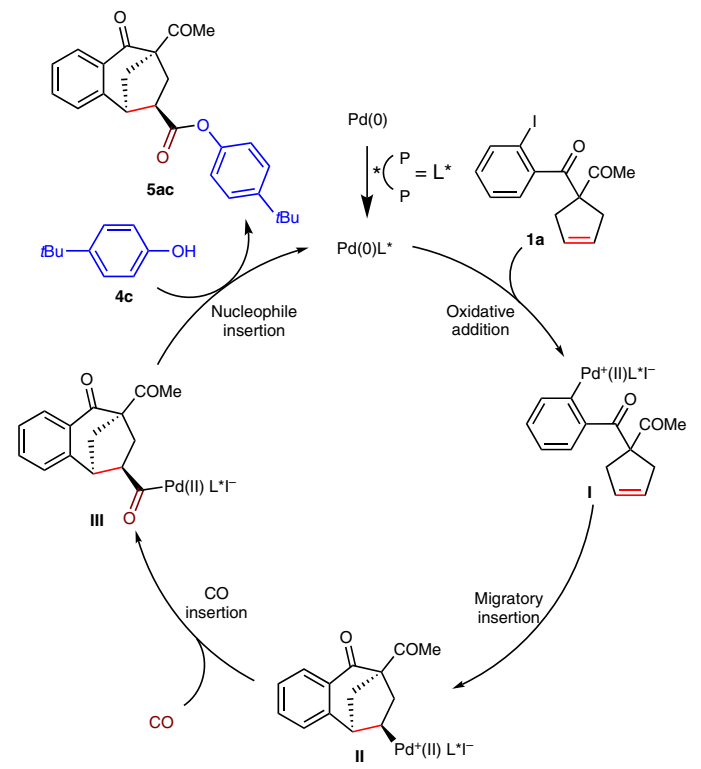

b

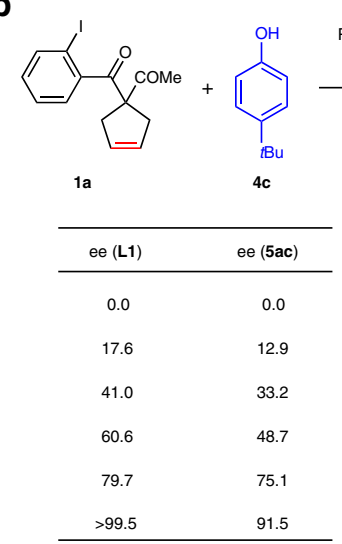

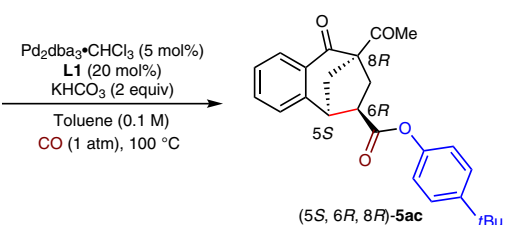

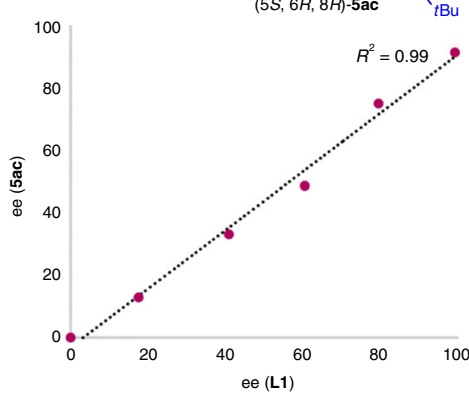

d

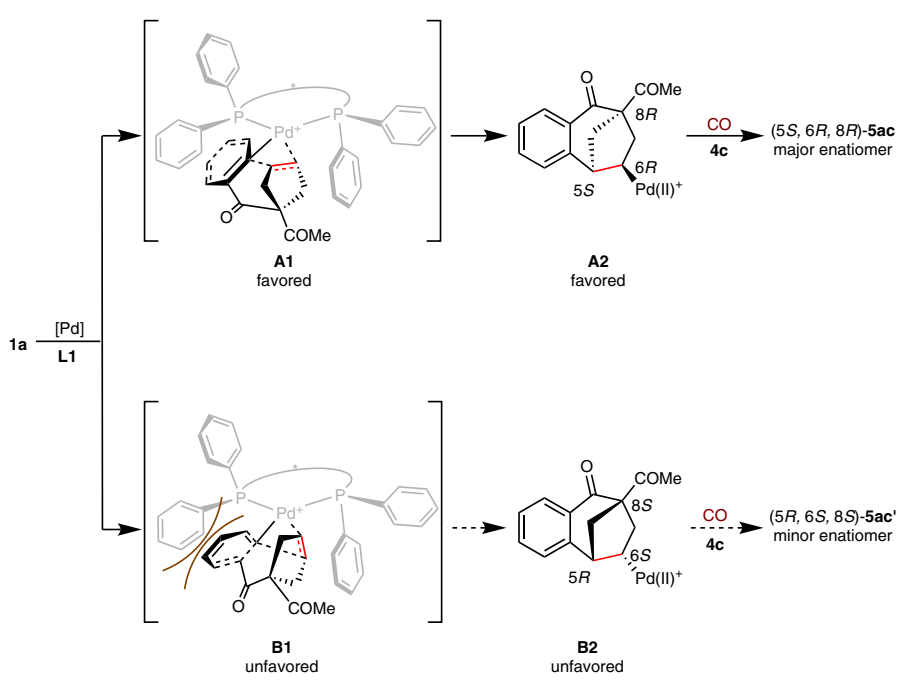

Fig. 5 Further study on the reaction. a Enantiodivergent synthesis of $(5 R, 6 S, 8 S)-\mathbf{5 a c} \mathbf{c}^{\prime}$. b Linear correlation between the ee values of $\mathbf{5 a c}$ and $\mathbf{L 1}$ c Proposed mechanism. d Model for enantioselectivity.

\section{Data availability}

The authors declare that all the data supporting the findings of this study are available within the article and Supplementary Information files, and are also available from the corresponding author upon reasonable request. The X-ray crystallographic coordinates for structures reported in this article have been deposited at the Cambridge Crystallographic Data Center (CCDC) under deposition numbers 1936197 (3aa) and 1936198 (7aa). These data could be obtained free of charge from The Cambridge Crystallographic Data Center via http://www.ccdc.cam.ac.uk/data_request/cif.

Received: 1 January 2020; Accepted: 16 April 2020;

Published online: 21 May 2020

\section{References}

1. Brennfuehrer, A., Neumann, H. \& Beller, M. Palladium-catalyzed carbonylation reactions of aryl halides and related compounds. Angew. Chem. Int. Ed. 48, 4114-4133 (2009).

2. Liu, Q., Zhang, H. \& Lei, A. Oxidative carbonylation reactions: organometallic compounds (R-M) or hydrocarbons (R-H) as nucleophiles. Angew. Chem. Int. Ed. 50, 10788-10799 (2011).

3. Sumino, S., Fusano, A., Fukuyama, T. \& Ryu, I. Carbonylation reactions of alkyl iodides through the interplay of carbon radicals and Pd catalysts. Acc. Chem. Res. 47, 1563-1574 (2014).

4. Wu, X.-F. et al. Transition-metal-catalyzed carbonylation reactions of Olefins and alkynes: a personal account. Acc. Chem. Res. 47, 1041-1053 (2014).

5. $\mathrm{Wu}, \mathrm{L}$. et al. Palladium-catalyzed carbonylative transformation of $\mathrm{C}(\mathrm{sp} 3)-\mathrm{X}$ bonds. ACS Catal. 4, 2977-2989 (2014).
6. Chen, J. et al. Base-controlled selectivity in the synthesis of linear and angular fused quinazolinones by a palladium-catalyzed carbonylation/nucleophilic aromatic substitution sequence. Angew. Chem. Int. Ed. 53, 7579-7583 (2014).

7. Lin, M., Li, F., Jiao, L. \& Yu, Z.-X. Rh(I)-catalyzed formal $[5+1] /[2+2+1]$ cycloaddition of 1-Yne-vinylcyclopropanes and two CO units: one-step construction of multifunctional angular tricyclic 5/5/6 compounds. J. Am. Chem. Soc. 133, 1690-1693 (2011).

8. Li, X., Song, W. \& Tang, W. Rhodium-catalyzed tandem annulation and (5 $+1)$ cycloaddition: 3-hydroxy-1,4-enyne as the 5-carbon component. J. Am. Chem. Soc. 135, 16797-16800 (2013).

9. Cheng, J., Qi, X., Li, M., Chen, P. \& Liu, G. Palladium-catalyzed intermolecular aminocarbonylation of alkenes: efficient access of $\beta$-amino acid derivatives. J. Am. Chem. Soc. 137, 2480-2483 (2015).

10. Li, M., Yu, F., Qi, X., Chen, P. \& Liu, G. Cooperative strategy for the highly selective intermolecular oxycarbonylation reaction of alkenes using a palladium catalyst. Angew. Chem. Int. Ed. 55, 13843-13848 (2016).

11. Qi, X., Yu, F., Chen, P. \& Liu, G. Palladium-catalyzed intermolecular oxidative fluorocarbonylation of unactivated alkenes: efficient access of $\beta$ fluorocarboxylic esters. Angew. Chem. Int. Ed. 56, 12692-12696 (2017).

12. Ding, D., Zhu, G.-H. \& Jiang, X.-F. Ligand controlled Pd(II)-catalyzed regiodivergent carbonylation of alkynes for syntheses of indolo[3,2-c] coumarins and benzofuro[3,2-c]quinolinones. Angew. Chem. Int. Ed. 57, 9028-9032 (2018).

13. Wu, L.-J., Song, R.-J., Luo, S. \& Li, J.-H. Palladium-catalyzed reductive [5+1] cycloaddition of 3-acetoxy-1,4-enynes with CO: access to phenols enabled by hydrosilanes. Angew. Chem. Int. Ed. 57, 13308-13312 (2018).

14. Li, X., Chen, P. \& Liu, G. Pd-catalyzed intermolecular arylcarbonylation of unactivated alkenes: incorporation of bulky aryls at room temperature. Angew. Chem. Int. Ed. 57, 15871-15876 (2018). 
15. Zheng, W.-F. et al. Tetrasubstituted allenes via the palladium-catalysed kinetic resolution of propargylic alcohols using a supporting ligand. Nat. Catal. 2, 997-1005 (2019).

16. Yang, J. et al. Direct synthesis of adipic acid esters via palladium-catalyzed carbonylation of 1,3-dienes. Science 366, 1514-1517 (2019).

17. Negishi, E.-i et al. Palladium-catalyzed carbonylative cyclization of 1-iodo-2alkenylbenzenes. J. Am. Chem. Soc. 118, 5904-5918 (1996).

18. Aggarwal, V. K., Davies, P. W. \& Moss, W. O. A palladium catalysed cyclisation-carbonylation of bromodienes: control in carbonylation over facile $\beta$-hydride elimination. Chem. Commun. 9, 972-973 (2002).

19. Seashore-Ludlow, B. \& Somfai, P. Domino carbopalladation-carbonylation: generating quaternary stereocenters while controlling $\beta$-hydride elimination. Org. Lett. 12, 3732-3735 (2010).

20. Seashore-Ludlow, B., Danielsson, J. \& Somfai, P. Domino carbopalladationcarbonylation: investigation of substrate scope. Adv. Synth. Catal. 354, 205-216 (2012).

21. Liu, X. \& Gu, Z. Pd-catalyzed heck cyclization and in situ hydrocarboxylation or hydromethenylation via a hydrogen borrowing strategy. Org. Chem. Front. 2, 778-782 (2015).

22. Gehrtz, P. H., Hirschbeck, V., Ciszek, B. \& Fleischer, I. Carbonylations of alkenes in the total synthesis of natural compounds. Synthesis 48, 1573-1596 (2016).

23. Bai, Y., Davis, D. C. \& Dai, M. Natural product synthesis via palladiumcatalyzed carbonylation. J. Org. Chem. 82, 2319-2328 (2017).

24. Ma, K., Martin, B. S., Yin, X. \& Dai, M. Natural product syntheses via carbonylative cyclizations. Nat. Prod. Rep. 36, 174-219 (2019).

25. Artman, G. D. \& Weinreb, S. M. An approach to the total synthesis of the marine ascidian metabolite perophoramidine via a halogen-selective tandem Heck/carbonylation strategy. Org. Lett. 5, 1523-1526 (2003).

26. Bai, Y., Shen, X., Li, Y. \& Dai, M. Total synthesis of (-)-spinosyn A via carbonylative macrolactonization. J. Am. Chem. Soc. 138, 10838-10841 (2016).

27. Xu., L., Wang, C., Gao, Z. \& Zhao, Y.-M. Total Synthesis of ( \pm )-cephanolides $\mathrm{B}$ and $\mathrm{C}$ via a palladium-catalyzed cascade cyclization and late-stage sp3 $\mathrm{C}-\mathrm{H}$ bond oxidation. J. Am. Chem. Soc. 140, 5653-5658 (2018).

28. Ma, K., Yin, X. \& Dai, M. Total syntheses of bisdehydroneostemoninine and bisdehydrostemoninine by catalytic carbonylative spirolactonization. Angew. Chem. Int. Ed. 57, 15209-15212 (2018).

29. Han, A., Tao, Y. \& Reisman, S. E. A 16-step synthesis of the isoryanodane diterpene (+)-perseanol. Nature 573, 563-567 (2019).

30. Zanti, G. \& Peeters, D. DFT study of small palladium clusters Pdn and their interaction with a CO ligand $(n=1-9)$. Eur. J. Inorg. Chem. 2009, 3904-3911 (2009).

31. Noonan, G. M. et al. Regioselective and enantioselective hydroformylation of dialkylacrylamides. Adv. Synth. Catal. 352, 1047-1054 (2010).

32. Carmona, R. C., Koster, O. D. \& Duarte Correia, C. R. Chiral N,N ligands enabling palladium-catalyzed enantioselective intramolecular Heck-Matsuda carbonylation reactions by sequential migratory and CO insertions. Angew. Chem. Int. Ed. 57, 12067-12070 (2018).

33. $\mathrm{Hu}, \mathrm{H}$. et al. Enantioselective synthesis of 2-oxindole spiro-fused lactones and lactams by Heck/carbonylative cyclization: method development and applications. Angew. Chem. Int. Ed. 58, 9225-9229 (2019).

34. Cheng, C., Wan, B., Zhou, B., Gu, Y. \& Zhang, Y. Enantioselective synthesis of quaternary 3,4-dihydroisoquinolinones via Heck carbonylation reactions: development and application to the synthesis of Minalrestat analogues. Chem. Sci. 10, 9853-9858 (2019).

35. Filippini, M. H. \& Rodriguez, J. Synthesis of functionalized bicyclo[3.2.1]octanes and their multiple uses in organic chemistry. Chem. Rev. 99, 27-76 (1999).

36. Presset, M., Coquerel, Y. \& Rodriguez, J. Syntheses and applications of functionalized bicyclo[3.2.1] octanes: thirteen years of progress. Chem. Rev. 113, 525-595 (2013).

37. Bashore, C. G. et al. Enantioselective synthesis of nicotinic receptor probe 7,8difluoro-1,2,3,4,5,6-hexahydro-1,5-methano-3-benzazocine. Org. Lett. 8, 5947-5950 (2006).

38. Grachan, M. L., Tudge, M. T. \& Jacobsen, E. N. Enantioselective catalytic carbonyl-ene cyclization reactions. Angew. Chem. Int. Ed. 47, 1469-1472 (2008).

39. Rueping, M., Kuenkel, A., Tato, F. \& Bats, J. W. Asymmetric organocatalytic domino Michael/Aldol reactions: enantioselective synthesis of chiral cycloheptanones, tetrahydrochromenones, and polyfunctionalized bicyclo[3.2.1] octanes. Angew. Chem. Int. Ed. 48, 3699-3702 (2009).

40. Huwyler, N. \& Carreira, E. M. Total synthesis and stereochemical revision of the chlorinated sesquiterpene ( \pm )-gomerone C. Angew. Chem. Int. Ed. 51, 13066-13069 (2012).

41. Zhu, S., Zhang, Q., Chen, K. \& Jiang, H. Synergistic catalysis: metal/protoncatalyzed cyclization of alkynones toward bicyclo[3.n.1]alkanones. Angew. Chem. Int. Ed. 54, 9414-9418 (2015).

42. Ando, Y., Hori, S., Fukazawa, T., Ohmori, K. \& Suzuki, K. Toward naphthocyclinones: doubly connected octaketide dimers with a bicyclo[3.2.1] octadienone core by thiolate-mediated cyclization. Angew. Chem. Int. Ed. 54, 9650-9653 (2015).
43. Burns, A. R., Madec, A. G. E., Low, D. W., Roy, I. D. \& Lam, H. W Enantioselective synthesis of bicyclo[3.n.1]alkanes by chiral phosphoric acidcatalyzed desymmetrizing Michael cyclizations. Chem. Sci. 6, 3550-3555 (2015).

44. Zhu, C., Wang, D., Zhao, Y., Sun, W.-Y. \& Shi, Z. Enantioselective palladiumcatalyzed intramolecular $\alpha$-arylative desymmetrization of 1,3-diketones. J. Am. Chem. Soc. 139, 16486-16489 (2017).

45. He, C., Hu, J., Wu, Y. \& Ding, H. Total syntheses of highly oxidized entkaurenoids pharicin A, pharicinin B, 7-O-acetylpseurata C, and pseurata C: A [5+2] cascade approach. J. Am. Chem. Soc. 139, 6098-6101 (2017).

46. Ramachary, D. B., Anif Pasha, M. \& Thirupathi, G. Organocatalytic asymmetric formal [3+2] cycloaddition as a versatile platform to access methanobenzo[7] annulenes. Angew. Chem. Int. Ed. 56, 12930-12934 (2017).

47. Zhao, L., Li, Z., Chang, L., Xu, J., Yao, H. \& Wu, X. Efficient construction of fused indolines with a 2-quaternary center via an intramolecular Heck reaction with a low catalyst loading. Org. Lett. 14, 2066-2069 (2012).

48. Su, Y., Zhou, H., Chen, J., Xu, J., Wu, X., Lin, A. \& Yao, H. Solvent-controlled switchable $\mathrm{C}-\mathrm{H}$ alkenylation of 4-aryl-1H-pyrrole-3-carboxylates: application to the total synthesis of ( \pm )-rhazinilam. Org. Lett. 16, 4884-4887 (2014).

49. Yuan, Z., Feng, Z., Zeng, Y., Zhao, X., Lin, A. \& Yao, H. Palladium-catalyzed asymmetric intramolecular reductive Heck desymmetrization of cyclopentenes: access to chiral bicyclo [3.2.1] octanes. Angew. Chem. Int. Ed. 58, 2884-2888 (2019).

50. Han, C., Fu, Z., Guo, S., Fang, X., Lin, A. \& Yao, H. Palladium-catalyzed remote 1,n-arylamination of unactivated terminal alkenes. ACS Catal. 9, 4196-4202 (2019).

51. Watson, D. A., Fan, X. \& Buchwald, S. L. Carbonylation of aryl chlorides with oxygen nucleophiles at atmospheric pressure. Preparation of phenyl esters as acyl Transfer agents and the direct preparation of alkyl esters and carboxylic acids. J. Org. Chem. 73, 7096-7101 (2008).

52. Wu, X.-F., Neumann, H. \& Beller, M. Convenient carbonylation of aryl bromides with phenols to form aryl esters by applying a palladium/di-1 adamantyl-n-butylphosphine catalyst. ChemCatChem 2, 509-513 (2010).

\section{Acknowledgements}

The authors thank the National Natural Science Foundation of China (NSFC21572272), the Innovation Team of "the Double-First Class" Disciplines (CPU2018GY04 and CPU2018GY35), and the Foundation of the Open Project of State Key Laboratory of Natural Medicines (SKLNMZZCX201818) for the financial support.

\section{Author contributions}

Z.Y. planned and conducted most of the experiments; Z.Y., Y.Z., Z.F., and Z. G. prepared substrates for the reaction scope evaluation; A.L. and H.Y. directed the projects and cowrote the manuscript. All authors contributed to the discussion.

\section{Competing interests}

The authors declare no competing interests.

\section{Additional information}

Supplementary information is available for this paper at https://doi.org/10.1038/s41467 020-16221-9.

Correspondence and requests for materials should be addressed to A.L. or H.Y.

Peer review information Nature Communications thanks Zhuangzhi Shi and the other, anonymous, reviewer(s) for their contribution to the peer review of this work.

Reprints and permission information is available at http://www.nature.com/reprints

Publisher's note Springer Nature remains neutral with regard to jurisdictional claims in published maps and institutional affiliations.

Open Access This article is licensed under a Creative Commons Attribution 4.0 International License, which permits use, sharing, adaptation, distribution and reproduction in any medium or format, as long as you give appropriate credit to the original author(s) and the source, provide a link to the Creative Commons license, and indicate if changes were made. The images or other third party material in this article are included in the article's Creative Commons license, unless indicated otherwise in a credit line to the material. If material is not included in the article's Creative Commons license and your intended use is not permitted by statutory regulation or exceeds the permitted use, you will need to obtain permission directly from the copyright holder. To view a copy of this license, visit http://creativecommons.org/licenses/by/4.0/. 